\title{
Examining a processing tradeoff explanation of proactive interference
}

\author{
ELLIOT HIRSHMAN \\ University of North Carolina, Chapel Hill, North Carolina \\ DANIEL J. BURNS \\ Lafayette College, Easton, Pennsylvania \\ and \\ TZY-MEY KUO \\ University of North Carolina, Chapel Hill, North Carolina
}

\begin{abstract}
Burns (1989) claims that proactive interference effects occur in paired-associate learning because of tradeoffs in relational and response-specific processing. Consistent with this claim, Burns demonstrated that free recall of critical-list responses is better in the interference condition than in the control condition. Burns's processing tradeoff explanation predicts that the occurrence of this reverse-interference effect should be positively correlated with the occurrence of traditional interference effects. We present several experiments whose results are inconsistent with this prediction. We hypothesize that the reverse-interference effect is a list-length effect. The results of a final experiment, contrasting the predictions of the list-length and processing tradeoff explanations, support the list-length explanation.
\end{abstract}

Understanding the theoretical mechanisms of interference effects has engaged learning and memory theorists for decades (see Crowder, 1976, for an extensive review). Burns (1989) has recently presented an alternative framework for understanding proactive interference in pairedassociate learning. Burns claims that proactive interference arises in paired-associate learning because subjects employ different encoding strategies in the control (D-B, A-C) and interference (A-B, A-C) conditions during second-list $(\mathrm{A}-\mathrm{C})$ learning. Burns characterizes these encoding strategies using the distinction between relational and item-specific processing (Hunt \& Einstein, 1981). Burns claims that subjects in the interference condition focus on response-specific information, at the expense of relational information, during the encoding of the second-list $(\mathrm{A}-\mathrm{C})$ pairs. This deficit in relational processing is assumed to cause proactive interference in paired-associate learning. One motivation for this processing tradeoff may be that subjects in the interference condition realize that stimulus-response mediators have been "used up" during prior-list learning.

Burns's (1989) position predicts that interference effects should reverse on memory tests that are sensitive primarily to the memorial characteristics of response terms. Consistent with this prediction, Burns demonstrated that free

\footnotetext{
The authors thank Margaret Intons-Peterson and several anonymous reviewers for helpful comments during the course of the current research. Correspondence concerning this article should be addressed to E. Hirshman, Department of Psychology, CB $\$ 3270,206$ Davie Hall, University of North Carolina at Chapel Hill, Chapel Hill, NC 27599-3270, or D. J. Burns, Department of Psychology, Lafayette College, Easton, PA 18042.
}

recall of second-list responses was better in the interference condition than in the control condition. We will call this finding a reverse-interference effect. In addition to supporting Burns's position, the reverse-interference effect provides problems for previous accounts of proactive interference (Gardiner, Craik, \& Birtwistle, 1972; Greeno, James, \& DaPolito, 1971; Postman \& Underwood, 1973). This is because these accounts cannot explain any memorial advantage for the interference condition.

This paper focuses on a second prediction of Burns's (1989) framework. Burns's position predicts that traditional interference effects and reverse-interference effects should be positively correlated. That is, manipulations that increase traditional interference effects should increase reverse-interference effects, and manipulations that remove traditional interference effects should remove reverse-interference effects. This prediction arises because the same mechanism, the hypothesized tradeoff between relational and response-specific processing, is assumed to cause both effects.

In this paper, we present several experiments that demonstrate the independence of traditional interference and reverse-interference effects. These findings are inconsistent with Burns's (1989) account of proactive interference effects. We consider an alternative explanation of reverseinterference effects for which we provide empirical support.

\section{EXPERIMENTS 1 AND 2}

Experiments 1 and 2 were designed to be conceptual replications of Burns's (1989) experiments. To our sur- 
prise, the results of both experiments demonstrated that reverse-interference effects occur independently of traditional interference effects. This finding raises questions about the viability of the processing tradeoff hypothesis.

\section{Method}

The methods of Experiments 1 and 2 were identical, except that the experimenters and subject populations differed

Subjects. The subjects in Experiment 1 were 30 undergraduates at Creighton University, who participated for extra credit in a psychology class. The subjects in Experiment 2 were 36 undergraduates at the University of North Carolina at Chapel Hill, who participated as part of an Introductory Psychology class requirement.

Design and Materials. Experiments 1 and 2 used a one-factor between-subject design with three dependent measures. Prior learning (control vs. interference) was manipulated between subjects. The three dependent measures were (1) cued recall of the prior (i.e., first) list; (2) cued recall of the critical (i.e., second) list; and (3) free recall of the critical-list responses.

Three lists containing 24 word pairs each were used. The items in the pairs were unrelated words selected from Toglia and Battig's (1978) materials (e.g., WALK-JUDGE, SLEEP-FLAG). The three lists were (1) the prior (i.e., D-B) list presented in the control condition; (2) the prior (i.e., A-B) list presented in the interference condition; and (3) the critical (i.e., A-C) list that was presented in both the control and interference conditions. Pairs in the prior lists in the control and interference conditions share response terms (i.e., D-B, A-B). Pairs in the prior and critical lists in the interference condition share stimulus terms (i.e., A-B, A-C).

Procedure. The subjects were tested in small groups; groups of subjects were randomly assigned to levels of the between-subjects factor. At the beginning of the experiment, the subjects were in formed that they would be presented with word pairs and that their memory task would be to recall the response term from a pair when given the stimulus term from the pair. Following these instructions, the 24 word pairs from the prior list were presented for $7 \mathrm{sec}$ each. The prior list differed in the interference and control conditions. The subjects in the interference condition received the A-B list, and the subjects in the control condition received the D-B list. Following the presentation of the prior list, the subjects were given a cued-recall test. In this test, they were presented with the stimulus terms from the study list and were asked to write the appropriate response term next to each stimulus term. Following the cuedrecall test, the critical list was presented. The procedures for the critical list were identical to those of the prior list, with two exceptions. First, following the cued-recall test, the subjects were asked to write down the names of states for $5 \mathrm{~min}$. Second, following this distractor task, they were given 5 min to freely recall the response terms from the critical list.

\section{Results}

Experiment 1. The proportion correctly recalled in cued recall of the prior list did not differ between the control and interference conditions (.45 vs. $.48, F<1)$. Thus, prior learning is approximately equal across the two experimental conditions. The proportion correctly recalled in cued recall of the critical list did not differ between the control and interference conditions ( .62 vs. .62, $F<$ 1). This represents a failure to replicate traditional interference effects. The proportion correctly recalled in free recall of critical-list responses was better in the interference condition than in the control condition [.34 vs. .21, $\left.F(1,28)=5.85, M S_{\mathrm{e}}=.022, p<.05\right]$. This replicates Burns's (1989) reverse-interference effect.
Experiment 2. The results of Experiment 2 were identical to those of Experiment 1. Cued recall of the prior list did not differ significantly between the control and interference conditions (.42 vs. $.45, F<1)$. Cued recall of the critical list did not differ between the control and interference conditions $(.57$ vs. $.56, F<1)$. Free recall of critical-list responses was better in the interference condition than in the control condition [.41 vs. .22, $\left.F(1,34)=9.87, M S_{\mathrm{e}}=.032, p<.01\right]$.

\section{Discussion}

Experiments 1 and 2 demonstrate that reverseinterference effects occur in free recall when traditional interference effects do not occur in cued recall. This finding seems inconsistent with Burns's (1989) processing tradeoff hypothesis. Burns's hypothesis claims that greater response-specific encoding is accompanied by less relational encoding in the interference condition. These differences lead to traditional and reverse-interference effects, respectively. Thus, finding reverse-interference effects without traditional interference effects is puzzling for the processing tradeoff hypothesis.

It is, however, possible to reconcile the results of Experiments 1 and 2 with Burns's (1989) hypothesis by focusing on the sensitivity of traditional and reverseinterference effects to differences in underlying memory representations. In this view, the results of Experiments 1 and 2 arise because a greater difference in underlying representations is required to produce traditional interference effects than to produce reverse-interference effects. When only a single trial is given for prior-list learning, the processing tradeoff produces a difference in memory that is sufficient to produce a reverse-interference effect, but not a traditional interference effect.

Experiment 3 tests this alternative interpretation by presenting the prior list for three, instead of one, study-test trials. This should substantially increase the differences between the memory representations of the interference and control conditions (i.e., the stronger prior-list associations will lead to a greater processing tradeoff). According to the preceding interpretation, this should produce both traditional and reverse-interference effects in Experiment 3 . This finding would be consistent with the classic finding (Underwood \& Ekstrand, 1966) that increasing prior-list learning increases traditional interference effects.

\section{EXPERIMENT 3}

\section{Method}

The method of Experiment 3 was identical to that of Experiments 1 and 2 with two exceptions. First, 60 subjects from Creighton University and Lafayette College participated in Experiment 3. Second, the prior list was presented for three study-test trials instead of one.

\section{Results and Discussion}

Prior-list learning. We conducted a $3 \times 2$ mixed analysis of variance (ANOVA) using the factors of prior- 
learning trial (Trial 1 vs. Trial 2 vs. Trial 3) and priorlearning condition (control vs. interference) to determine if prior learning differed across the control and interference conditions. It did not. Our analysis produced one significant effect. Cued-recall performance improved across trials $[.49$ vs. .82 vs. $.94, F(2,116)=276.15$, $\left.M S_{\mathrm{e}}=.012, p<.001\right]$. The high level of performance on Trial 3 indicates that prior-list learning produced strong associations in the prior-list pairs. This high level of priorlist learning contrasts to the lower levels of learning in Experiments 1 and 2 (.47 and .44, respectively).

Critical-list performance. Cued-recall performance was better in the control condition than in the interference condition $\left[.70\right.$ vs. $.56, F(1,58)=5.63, M S_{e}=.05$, $p<.05]$. This replicates the traditional interference effect. Response free recall was better in the interference condition than in the control condition [.43 vs. .29 , $\left.F(1,58)=6.55, M S_{\mathrm{e}}=.044, p<.05\right]$. This replicates the reverse-interference effect.

The results of Experiment 3 demonstrate both traditional and reverse-interference effects, thus replicating Burns's (1989) results. When prior-list associations are sufficiently strong, traditional interference effects, as well as reverse-interference effects, occur. This result suggests that the failure to demonstrate traditional interference effects in Experiments 1 and 2 may have occurred because traditional interference effects are less sensitive to differences in underlying memory representations than reverseinterference effects. Under this interpretation, the processing tradeoff hypothesis is still viable.

Accordingly, Experiment 4 was designed to provide an additional test of the processing tradeoff hypothesis. In Experiment 4 , we manipulated the preexperimental strength of prior-list pairs (strong vs. unrelated), as well as the priorlearning condition (control vs, interference). For some subjects, the items in the prior-list pairs were strongly associated (e.g., TABLE-CHAIR), whereas for other subjects, the items were unrelated (e.g., DOG-CHAIR). The procedures of Experiment 4 were identical to those of Experiments 1 and 2 with the notable exception that half the subjects received strongly related, as opposed to unrelated, pairs in the prior list.

We think this design provides an appropriate test of the processing tradeoff hypothesis because, unlike the designs of Experiments 1 and 2, it provides a direct test of whether the occurrence of traditional interference and reverseinterference effects are correlated. Specifically, if strongly associated prior lists enhance traditional interference effects, a result predicted by the results of Experiments 1-3, then the processing tradeoff hypothesis postulates that this manipulation should also enhance reverse-interference effects. This is because the processing tradeoff hypothesis would have to posit a greater tradeoff in the processing of relational and response-specific information to account for enhanced traditional interference effects, leading to a prediction of larger reverse-interference effects. Thus, Experiment 4 tests the processing tradeoff hypothesis by seeing if both traditional and reverse-interference effects are enhanced by strongly associated prior lists.

\section{EXPERIMENT 4}

\section{Method}

The method of Experiment 4 was identical to that of Experiments 1 and 2 with three exceptions. First, the subjects were 112 undergraduates at Lafayette College who participated in the experiment for extra credit in a psychology course. Second, the experiment orthogonally manipulated prior-list associative strength (strong vs. unrelated) and prior learning (control vs. interference) between subjects. Third, the materials were drawn from Tulving and Thomson's (1973) materials. For the strongly associated prior-list condition, Tulving and Thomson's List A pairs were presented in the interference condition's prior list, and their List B pairs were presented in the control condition's prior list. ${ }^{2}$ For the unrelated priorlist condition, the stimulus-response pairs from the comparable lists (i.e., control and interference) of the strongly associated prior-list condition were randomly repaired. The critical-list materials for all four conditions consisted of stimulus terms from Tulving and Thomson's List A, paired with unrelated responses.

\section{Results and Discussion}

Prior-list learning. We conducted a $2 \times 2$ ANOVA using the between-subjects factors of prior-list associative strength (strong vs. unrelated) and prior-learning condition (control vs. interference) on the prior-learning scores to determine if prior learning differed across the experimental conditions. This analysis produced one significant effect. Prior learning was greater for strongly associated than for unrelated pairs $[F(1,108)=208.9$, $M S_{e}=.031, p<.001, .97$ vs. .48]. This indicates, as one would expect, that prior-list associations are stronger for related than for unrelated pairs.

Critical-list performance: Cued recall. We conducted a $2 \times 2$ ANOVA using the between-subjects factors of prior-list associative strength (strong vs. unrelated) and prior-learning condition (control vs. interference) on the cued-recall scores to determine if critical-list cued recall differed across the experimental conditions. This analysis produced one effect of interest. The interaction of prior-list associative strength and prior learning approached the traditional criterion for significance $\left[F(1,108)=3.06, M S_{\mathrm{e}}=.044, p=.08\right]$. Table 1 presents the means for the four experimental conditions.

The means in Table 1 present two important results. First, as in Experiments 1 and 2, traditional interference effects do not occur when unrelated pairs are presented for a single prior-learning trial (control $=.55$, interference $=.56$ ). Second, traditional interference effects occur when strongly associated pairs are used in the prior list (control $=.58$, interference $=.45$ ). Planned comparisons confirmed these observations. Performance in the control condition equaled performance in the interference

Table 1

Critical-List Cued-Recall Performance as a Function of Prior-List Associative Strength and Prior Learning

\begin{tabular}{lcc} 
& \multicolumn{2}{c}{ Associative Strength } \\
Prior Learning & Strongly Associated & Unrelated \\
Control & .58 & .55 \\
Interference & .45 & .56
\end{tabular}


Table 2

Critical-List Free-Recall Performance as a Function of Prior-List Associative Strength and Prior Learning

Associative Strength

\begin{tabular}{lcc} 
Prior Learning & Strongly Associated & Unrelated \\
\hline Control & .33 & .30 \\
Interference & .30 & .40
\end{tabular}

condition when unrelated pairs were used in prior learning $(F<1)$, but performance in the control condition was superior to performance in the interference condition when strongly associated pairs were used in prior learning $[F(1,54)=5.35, p<.05]$. As in our comparison of the results of Experiments 1-3, these results indicate that traditional interference effects are enhanced when priorlist associations are stronger. The processing tradeoff hypothesis predicts the same pattern for reverse-interference effects.

Critical-list performance: Free recall. The results in critical-list free recall do not show this pattern. We conducted a $2 \times 2$ ANOVA using the between-subjects factors of prior-list associative strength (strong vs. unrelated) and prior-learning condition (control vs. interference) on the free-recall scores to determine if critical-list free recall differed across the experimental conditions. This analysis produced one significant effect. The effect of priorlist associative strength interacted with the effect of priorlearning condition $\left[F(1,108)=7.79, M S_{\mathrm{e}}=.015, p<\right.$ $.05]$. Table 2 presents the means for the four experimental conditions.

The means in Table 2 present two important results. First, as in Experiments 1 and 2, reverse-interference effects occur when unrelated pairs are presented for a single prior-learning trial (control $=.30$, interference $=$ .40). Second, and most important, reverse-interference effects do not occur when strongly associated pairs are used in the prior list (control $=.33$, interference $=.30$ ). This result is inconsistent with the processing tradeoff hypothesis, which predicted that the reverse-interference effect would be enhanced when strongly associated priorlist pairs were used. In fact, the opposite result occurred. With strongly associated prior-list pairs, the reverseinterference effect did not occur. Planned comparisons confirmed the preceding observations. Performance in the interference condition was superior to performance in the control condition when unrelated prior-list pairs were used $[F(1,54)=10.33, p<.005]$, but performance in these two conditions was equal when strongly associated priorlist pairs were used $(F<1)$.

Further explorations of the reverse-interference effect. The results of the preceding studies demonstrate that the processing tradeoff hypothesis is not a sufficient explanation of both traditional and reverse-interference effects. It still, however, may be a viable explanation for one of the two effects. Because response competition (McGeoch, 1942) can explain the vicissitudes of tradi- tional interference effects in our experiments, we think it is unparsimonious to use the processing tradeoff hypothesis to explain traditional interference effects. On the other hand, the reverse-interference effect seems theoretically anomalous without the processing tradeoff hypothesis. Consequently, we focus here on whether the processing tradeoff hypothesis is a sufficient explanation of the reverse-interference effect. We do this by contrasting the processing tradeoff explanation to an alternative explanation of the reverse-interference effect. We first present this alternative explanation and then present an experiment that tests the contrasting predictions of the two explanations.

The alternative explanation conceptualizes the reverseinterference effect as a list-length effect. List-length effects (Roberts, 1972) occur when the probability of recalling items from a list decreases as the number of items on the list increases. The explanation is based on two assumptions. First, following Underwood and Ekstrand (1967), we assume that the representations of the prior and critical lists are intermingled in memory. For example, individual items from the prior list (e.g., A, B, or D terms) can be associated with the critical list's memory representation. Second, following Shiffrin, Ratcliff, and Clark (1990, pp. 182, 191), we assume that an item that is encoded repeatedly as a list item can be represented as a single list item in memory (i.e., multiple encodings of an item accrue strength to a single, common memory representation).

Here we use these assumptions to derive the reverseinterference effect. Under the first assumption, some prior-list items are encoded as critical-list items. Under the second assumption, these list differentiation failures increase the encoded or functional length of the critical list less in the interference condition than in the control condition. This is because A terms from the interference condition's prior list can be encoded with A terms from the interference condition's critical list as single criticallist items. This cannot occur in the control condition because there are no shared items between the prior and critical lists. This difference in the functional length of the critical list implies that the probability of recalling items, such as response terms, will be greater in the interference condition than in the control condition. This is the reverse-interference effect.

This list-length explanation provides a post hoc explanation for why reverse-interference effects disappear in Experiment 4 when strongly associated pairs are used in the prior list. We can conceptualize strongly associated pairs as single or unitized entities (Horowitz \& Prytulak, 1969) in memory. In this case, there will be no differences in the functional length of the critical list across the interference and control conditions. This is because the incorporation of the prior list's A terms into unitized pairs means that there are no functionally identical items in the interference condition's prior and critical lists. This means that list discrimination failures will increment the critical 
list's functional length equally in the control and interference conditions. Consequently, there will be no reverseinterference effect.

The list-length and processing tradeoff explanations generate contrasting predictions on whether the reverseinterference effect should occur for prior-list responses. The list-length explanation predicts that the reverseinterference effect should occur for prior-list responses. This prediction arises because the explanation for why the reverse-interference effect occurs for critical-list responses also applies to prior-list responses. Critical-list items can be encoded as prior-list items. These list differentiation failures will increase the functional length of the prior list more in the control condition than in the interference condition. This difference in functional list length will produce better recall of prior-list responses in the interference condition.

In contrast, the processing tradeoff hypothesis predicts that the reverse-interference effect should not occur for prior-list responses. This prediction arises because the processing tradeoff only occurs during critical-list processing. Experiment 5 tests these contrasting predictions.

\section{EXPERIMENT 5}

\section{Method}

The method of Experiment 5 was identical to that of Experiments 1 and 2 with two exceptions. First, the subjects were 54 undergraduates at Lafayette College who participated in the experiment for extra credit in a psychology course. Second, the subjects were asked to free recall the response terms from the prior list instead of the response terms from the critical list.

\section{Results and Discussion}

Cued recall of the prior list did not differ between the control and interference conditions ( .30 vs. $.34, F<1)$. Cued recall of the critical list did not differ between the control and the interference conditions ( .49 vs. . $42, F<1$ ). As in Experiments 1, 2, and 4, traditional interference effects did not occur when normatively unrelated pairs were presented for a single study-test trial.

Critically, a reverse-interference effect occurred for prior-list responses. Free recall of prior-list responses was better in the interference condition than in the control condition $\left[.22\right.$ vs. $.12, F(1,52)=7.89, M S_{\mathrm{e}}=.018, p<$ $.01]$. This result discriminates the list-length and processing tradeoff hypotheses. The list-length hypothesis correctly predicts reverse-interference effects for prior-list responses. This prediction arises because the explanation for why reverse-interference effects occur for critical-list responses also applies to prior-list responses. For either the critical or prior list, functional list length is greater in the interference condition than the control condition. In contrast, the processing tradeoff hypothesis incorrectly predicts a null effect for prior-list responses. This is because the processing tradeoff only occurs during criticallist learning. Thus, the processing tradeoff hypothesis is not a sufficient explanation for the reverse-interference effect. The list-length hypothesis remains a viable possi- bility and we will discuss it further in the General Discussion.

\section{GENERAL DISCUSSION}

Burns (1989) hypothesized that tradeoffs in relational and response-specific processing are responsible for proactive interference effects in paired-associate learning. In support of this view, Burns demonstrated that interference effects reverse in response free recall. Burns's view also predicts that the occurrence of reverseinterference effects should be positively correlated with the occurrence of traditional interference effects, because the same processing tradeoff is assumed to underlie both effects.

The results of our experiments were not consistent with the processing tradeoff hypothesis. Experiments 1 and 2 provided preliminary evidence that reverse-interference effects could occur independently of traditional interference effects. Experiment 4 demonstrated that a manipulation that removed the reverse-interference effect actually enhanced the traditional interference effect. These results demonstrate, contrary to the processing tradeoff hypothesis, that reverse-interference effects are not necessarily correlated with traditional interference effects. The results of Experiment 5 demonstrate that the processing tradeoff hypothesis is not even a sufficient explanation for the reverse-interference effect. Experiment 5 demonstrated that, contrary to the prediction of the processing tradeoff hypothesis, reverse-interference effects occur in prior-list response recall.

We considered the alternative hypothesis - that the reverse-interference effect is a list-length effect. The ideas motivating this conception are (1) failures in list discrimination increase the functional length of the critical list and (2) this increase is less in the interference condition than in the control condition because the $\mathrm{A}$ term that is repeated in the interference condition's prior and critical lists can be coded as a single item. These ideas imply that the functional length of the critical list is greater and, consequently, response free recall is worse in the control condition than in the interference condition. The results of Experiment 5, demonstrating reverse-interference effects in the free recall of prior-list responses, supported the listlength hypothesis.

The list-length explanation emphasizes two important aspects of memory. First, it describes another example of list-length effects. This emphasizes that any comprehensive model of memory must be able to explain these effects. (See Raaijmakers \& Shiffrin, 1981, for a model that can do this.) Second, by conceptualizing the repetition of the interference condition's A term as a single item in memory, the list-length hypothesis emphasizes the possibility that functional coding can have a counterintuitive relation to nominal stimuli.

Because the list-length hypothesis remains a viable explanation for the reverse-interference effect, we close by considering how it might relate to some other aspects of 
our results. We have argued that the list-length hypothesis can explain the null effect in critical-list response recall (Experiment 4) given the ancillary assumption that the strongly associated prior-list pairs have a unitized memory representation. Given this assumption, there are no functionally identical items in the interference condition's prior and critical lists and list discrimination failures will increment the length of the critical-list length equally in the interference and control conditions.

An important question for this elaborated account concerns how it explains the traditional interference effect found in Experiment 4. We answer this here in the context of response-competition conceptions of interference (McGeoch, 1942). In terms of response-competition theories, we can ask why the unitized A-B prior-list pair provides competition for the $C$ term from the $A-C$ pair when the $A$ term is presented as a cue. If we assume, as seems plausible from the data on A-B cued recall, that there is a strong association between the $A$ term as a cue and the unitized A-B pair stored in memory, then this unitized pair will provide response competition for the $C$ term just like any other item that is associated with the A term. This response competition will produce the traditional interference effect found in Experiment 4.

A second question concerns why reverse-interference effects occur in Experiment 3, when they do not occur in Experiment 4. After all, the results from prior-list cued recall in Experiment 3 indicate that the prior-list pairs are strongly associated by the end of the third study trial. If this strong association indicates unitization in memory, then the arguments presented above seem to imply that there should be no reverse-interference effect in Experiment 3. To understand why there is a reverse-interference effect, remember that while the prior-list pairs may be functionally unitized by the end of the third study trial, this is not the case on the first two study-test trials, nor at the beginning of the third study trial. Thus, as in Experiments $1,2,4$, and 5 , list discrimination failures, in which items presented on the prior list's study trials become part of the critical list's memory representation, will increment the length of the critical list more in the control condition than in the interference condition. As before, this will produce a reverse-interference effect.

These elaborations of the list-length hypothesis demonstrate its viability. An important task for further research would be to compare the list-length hypothesis to other possible explanations of the reverse-interference effect.

\section{REFERENCES}

Burns, D. J. (1989). Proactive interference: An individual item versus relational processing account. Journal of Memory \& Language, 28 , 345-359.

CROWDer, R. G. (1976). Principles of learning and memory. Hillsdale, NJ: Erlbaum.

Gardiner, J. M., Craik, F. I. M., \& BirTwistle, J. (1972). Retrieval cues and release from proactive inhibition. Journal of Verbal Learning \& Verbal Behavior, 11, 778-783

Greeno, J. G., James, C. T., \& DaPolito, F. J. (1971). A cognitive interpretation of negative transfer and forgetting of paired associates. Journal of Verbal Learning \& Verbal Behavior, 10, 331-345.

Horowitz, L. J., \& Prytulak, L. S. (1969). Redintegrative memory. Psychological Review, 76, 519-531.

Hunt, R. R., Einstein, G. O. (1981). Relational and item-specific information in memory. Journal of Verbal Learning \& Verbal Behavior, 20, 497-514.

McGeOCH, J. A. (1942). The psychology of human learning. New York, NY: Longmans.

Postman, L., \& Underwood, B. J. (1973). Critical issues in interference theory. Memory \& Cognition, 1, 19-40.

RaAiJmakers, J. G. W., \& ShIFfrin, R. M. (1981). Search of associative memory. Psychological Review, 88, 93-134.

RoBERTS, W. A. (1972). Free recall of word lists varying in length and presentation: A test of the total time hypothesis. Journal of Experimental Psychology, 92, 365-372.

Shiffrin, R. M., Ratcliff, R., \& Clark, S. E. (1970). List strength effect: II. Theoretical mechanisms. Journal of Experimental Psychology: Learning, Memory, \& Cognition, 16, 179-195.

Toglia, M. P., \& Battig, W. (1978). Handbook of semantic word norms. Hillsdale, $\mathrm{NJ}$ : Erlbaum.

Tulving, E., \& Thomson, D. M. (1973). Encoding specificity and retrieval processes in episodic memory. Psychological Review, 80, 352-373.

Underwood, B. J., \& Ekstrand, B. R. (1966). An analysis of some shortcomings in the interference theory of forgetting. Journal of Experimental Psychology, 73, 540-549.

UNDERWOOD, B. J. \& EKSTRAND, B. R. (1967). Studies of distributed practice XXIV: Differentiation and proactive inhibition. Journal of Experimental Psychology, 74, 574-580.

\section{NOTES}

1. Note that there is no evidence from Experiments 1, 2, or 3 that reverse-interference effects are enhanced when traditional interference effects are enhanced.

2. Note that this material's construction implies that our design compares performance in an A-B, A-C interference condition with performance in a D-E, A-C control condition. We used this control condition rather than the traditional D-B, A-C condition because it is difficult to find D-B pairs that are exeptionally strong associates when the A-B pairs are exceptionally strong associates.

(Manuscript received June 12, 1991; revision accepted for publication May 19, 1992.) 